\title{
Structure, signaling and the drug discovery of the Ras oncogene protein
}

\author{
Chang Woo Han, Mi Suk Jeong \& Se Bok Jang* \\ Department of Molecular Biology, College of Natural Sciences, Pusan National University, Busan 46241, Korea
}

\begin{abstract}
Mutations in Ras GTPase are among the most common genetic alterations in human cancers. Despite extensive research investigating Ras proteins, their functions still remain a challenge over a long period of time. The currently available data suggests that solving the outstanding issues regarding Ras could lead to development of effective drugs that could have a significant impact on cancer treatment. Developing a better understanding of their biochemical properties or modes of action, along with improvements in their pharmacologic profiles, clinical design and scheduling will enable the development of more effective therapies. [BMB Reports 2017; 50(7): 355-360]
\end{abstract}

\section{INTRODUCTION}

Ras was identified during the extensive study of retroviral oncogenes isolated from the genome of Harvey and Kirsten rat sarcoma viruses. Since the 1980s, the finding of mutated Ras genes in human tumor cell lines has led to intensive research into the structure and biochemistry of Ras (1). Ras proteins are small GTPases that serve as master regulators of countless signaling cascades involved in particularly diverse cellular processes. Activating mutations in Ras are found in about one-third of cancers. Oncogenic mutations in the Ras gene are related with a single mutation, typically at codons 12,13 or 61 (2). K-Ras mutations occur frequently in pancreatic, endometrial, colorectal, biliary tract, cervical, and lung cancers. $\mathrm{N}$-Ras and $\mathrm{H}$-Ras mutations prevail in melanoma and bladder cancer, respectively (3). Different isoforms of Ras $\left(\mathrm{H}_{-}, \mathrm{K}-\right.$-, and $\mathrm{N}$-Ras) can regulate a kind of cellular processes, including proliferation, differentiation, and apoptosis. Intensive efforts to target these $\mathrm{H}_{-}, \mathrm{K}-$, and $\mathrm{N}$-Ras key proteins have been conducted, but no efficient pharmacological inhibitors of the Ras proteins have been successfully applied in clinical settings.

${ }^{*}$ Corresponding author. Tel: +82-51-510-2523; Fax: +82-51-5812544; E-mail: sbjang@pusan.ac.kr

https://doi.org/10.5483/BMBRep.2017.50.7.062

Received 13 April 2017, Revised 9 May 2017, Accepted 28 May 2017
Recent development of new analysis tools in drug discovery has restarted our hope for development of a Ras inhibitor. However, Ras proteins are highly analogous in sequence and structure, especially in the catalytic domain, although important differences exist. The principal driver in most Ras-mutant cancers is K-Ras, but structural, biochemical and mutational data primarily originated in researches conducted using H-Ras (4). For these reasons, some possible binding sites have been confirmed employing computational accesses based on $\mathrm{H}$-Ras structural models; however, they do not seem to have any deep and hydrophobic patches on the surface of K-Ras that would permit strongly binding of small molecules (5). While the efforts to indirectly target Ras through FTIs were rationally designed, this strategy suffered from lack of consideration of the fundamental biology of Ras prenylation. This led to their subsequent failure in large-scale clinical trials targeting K-Ras mediated cancers (6). In previous studies, Ras effector signaling was considered to be a simple process. However, recently studies of various protein kinase cascades have revealed that Ras signaling occurs via a complicated and very dynamic signaling network that can adjust and resist in respond to inhibitors. Indiscriminately blocking Ras effectors for both wild type and mutant Ras may cause considerable toxicity. Hence, understanding Ras proteins can facilitate investigations of the interaction between development of cancer and cellular signaling pathways. In addition, understanding of the Ras structure has continuously improved since the first crystal structures of Ras were solved, leading to discovery of innovative and exciting venues for targeting inhibitors of Ras development (7). However, most of the inhibitors were ineffective because of low affinity and cellular toxicity. To solve this problem, recent studies have focused on downstream effectors that interact with Ras. These downstream effectors regulate the proliferation, survival, differentiation and motility of cancer cells through complex feedback and cross-talk mechanisms (8).

In this review, we suggest a deep analysis of the structure, activating mutations, signaling pathways, and inhibitors of Ras. We examine the problems associated with currently available Ras inhibitors and discuss hopeful means for additional development.

Keywords: Inhibitor, Mutation, Ras, Signaling

ISSN: 1976-670X (electronic edition)

Copyright (C) 2017 by the The Korean Society for Biochemistry and Molecular Biology

(c) This is an open-access article distributed under the terms of the Creative Commons Attribution Non-Commercial License (http://creativecommons.org/licenses/by-nc/4.0) which permits unrestricted non-commercial use, distribution, and reproduction in any medium, provided the original work is properly cited. 


\section{RAS STRUCTURE}

The Ras is Ras-related protein superfamily of small GTPbinding proteins with structural similarity (molecular weight 21-25 kDa) (9). Ras-related genes encoding mini GTP binding proteins fall into several subfamilies categorized by their amino acid sequences of encoded proteins and their biological functions, Ras, Rho, Rap and Ral (10). The Ras-related protein superfamily of small GTP-binding proteins is described by the supposed "G domain," which is distinct to this superfamily and plays mostly regulatory functions in many cellular processes. This domain, also called the switch I region (amino acids 32-40 in Ras), undergoes conformational changes during conversion of the guanosine diphosphate (GDP)-bound state into a guanosine triphosphate (GTP)-bound state. The Ras constitute a class of phosphate binding loop (P-loop) proteins that work as molecular switches between the GDP-bound inactive and the GTP-bound active state (11). The $\gamma$-phosphate interacts with key residues (Tyr32 and Thr35) that hold the switch I region. Conserved Gly60 of the switch II region (aa 59-77) makes crucial contacts with the $\gamma$-phosphate. The switch II region is located between the central $\beta$-sheet of Ras and the $\alpha 2$-helix (12).

The Ras genes, which are proto-oncogenes that are mutated in human cancers, are encoded by three expressed genes: $\mathrm{H}-$, $\mathrm{K}-$, and N-Ras (13). Three Ras genes encode 188-89 residue proteins that share about $82-90 \%$ total sequence identity. Ras proteins are processed in a series of reactions initiated by farnesylation of Ras. Although there are some striking differences in their primary structures, in particular in the variable carboxy terminal region, the enzyme farnesyltransferase recognizes the C-terminal sequence of the Ras gene known as the Cys-A-A-X motif, where A is isoleucine, leucine, or valine, and $X$ is serine or methionine (14). CAAX motif proteins play essential roles in multiple signaling pathways, controlling various processes (Fig. 1A). These reactions involve prenylation of the cysteine, split at the -AAX sequence and methylation of the carboxyl-prenylated cysteine residue (15). The -AAX sequence is removed by Ras Converting CAAX Endopeptidase 1 (Rce1) and the now $C$ farnesylated terminal cysteine is carboxyl methylated by isoprenylcysteine carboxyl methyltransferase (Icmt) (16). Hence, the CAAX motif comprises plasma membrane anchoring and trafficking of recently generalized Ras from the cytosolic surface of the endoplasmic reticulum (ER) to the internal surface of the plasma membrane (17). Plasma membrane anchoring and trafficking of Ras proteins circulate between an active GTP-bound state and an inactive GDP-bound state (Fig. 1B). These exchanges lead to large conformational changes of the switch I and II regions in the effector lobe of Ras. Their effector proteins consist Ras association (RA) domains or Ras binding domains (RBDs), which bind specifically to the GTP-bound state. Ras binds many effectors, which regulate signals over various cellular pathways (18).

\section{SIGNALING PATHWAY}

The anchored Ras proteins operate as molecular switches, which in the resting cells are in the GDP-bound inactive state. These Ras proteins become activated in response to extracellular receptors by binding GTP, as catalyzed by guanine nucleotide exchange factors (GEFs) son of sevenless 1 and 2 (SOS1 and SOS2) (19). In the GTP-bound active state, Ras interacts effectively with a series of cytoplasmic target or "effector" proteins (20) (Fig. 2). The Raf-MEK-ERK cascade is the most featured Ras effector pathway, getting suppression of

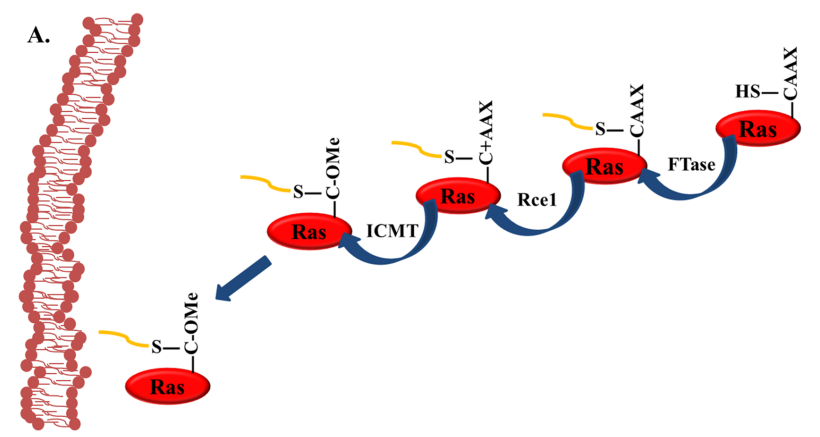

B.

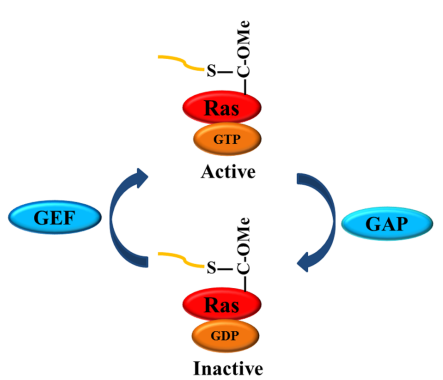

Fig. 1. Regulation of Ras membrane association. (A) Ras protein with a CAAX motif at the carboxyl terminus undergoes three posttranslational modifications (PTMs). The first modification step is addition of an isoprenyl group to the cysteine of the CAAX motif by farnesyltransferase (FTase). Next, the isoprenylated CAAX protein becomes a substrate for Ras converting enzyme 1 (RCE1), which removes the last three amino acids (the -AAX of the CAAX motif) by endoproteolysis. Finally, the newly exposed isoprenylated cysteine residue is methylated by isoprenylcysteine carboxyl methyltransferase (ICMT). (B) After trafficking and association with the inner face of the plasma membrane, Ras proteins cycle between inactive GDP-bound and active GTP-bound states. Growth factors stimulate transient activation of Ras through activation of GEF. Ras-GTP binds preferentially to downstream effectors. GAP accelerates the intrinsic GTP hydrolysis activity, returning Ras to the inactive state. 
cell growth, inhibition of cell death, invasiveness, and induction of angiogenesis (21). The initial mammalian effector of Ras to be featured was the Rapidly Accelerated Fibrosarcoma (Raf). Activated Ras functions as an adapter that binds to the serine/threonine-protein kinase with great affinity and generates translocation to the cell membrane, where Raf activation takes place (22). Downstream of this, activated Raf phosphorylates and activates mitogen-activated protein kinases 1 and 2 (MEK1 and MEK2), which can both activate the downstream mitogen-activated protein kinases (MAPKs) extracellular signal-regulated kinases 1 and 2 (ERK1 and ERK2) (23). They stimulate ERK1 and ERK2 through phosphorylation of a -Thr-Glu-Tyr- motif site in the activation loop. Notably, ERK $1 / 2$ is transcription factors that have a direct role in cell changing gene expression to enhance cellular growth, cell differentiation and cell division (24). In addition, ERKs can move to the nucleus and activate ETS family transcription factors, i.e. ternary complex factor (TCF) Elk-1, Ets1, serum response factor accessory protein Sap-1a, c-Myc, Tal etc. One of the Ras-induced cellular responses controls the expression of various genes, such as the prompt early gene c-fos, which enables the cell progression through G0/G1 mitogenic signals of the cell-cycle. As a result, Raf-MEK-ERK cascade activation can induce cell-cycle progression (25).

Ras has been identified to interact with and stimulate other signaling pathways that phosphatidylinositol 3-kinase (PI3K)phosphoinositide-dependent serine/threonine protein kinase (Akt)-mammalian target of rapamycin (mTOR) signaling (26). The PI3K-Akt-mTOR signaling pathway is crucial in signaling downstream of Ras as it regulates cell survival. Ras-PI3K controls the activity of the 3-phosphoinositide-dependent protein kinase-1 (PDK1) (27). PDK1 is a serine/threonine kinase belonging to protein kinases of the AGC kinase superfamily, including APK/PKB PKA, PKG and PKC (28). Akt/PBK regulates various cellular responses, including metabolism, angiogenesis, cell-growth, proliferation, survival, programmed cell-death, protein synthesis, and transcription by Ras-PI3K-PDK1 (29). In addition, PI3K-dependent activation of Ras-related C3 botulinum toxin substrate (RAC) regulates a broad range of generally crucial downstream responses, e.g. actin cytoskeleton, cell survival, cell/cell contacts and adhesion, transcription and translation etc. Activated RAC also induces ERK signaling and improves cellular sensitivity about growth factors (30).

Activated Ras has also been shown to stimulate the Ral specific guanine-nucleotide-exchange factors (Ral-GEFs). Ral guanine-nucleotide-exchange factors (RalGDS) belongs to family of guanine nucleotide exchange factors (GEFs) that promote activation of the Ras family member Ral, causing activation of phospholipase D1 (PLD1), an enzyme that regulates vesicle trafficking (31). In addition, Ral stimulation leads to activation of RALBP1 (also known as RLIP1 and RIP1). RALBP1 is a GTPase-activating protein (GAP) for CDC42 and Rac GTPases. One remarkable characteristic identified for RalBP1 was GAP activity for the Rho family GTPases Tac1 and Cdc42, therefore making RALBP1 the potential to affect actin dynamics and the formation of filopodia and membrane ruffling (32).

Phospholipase $\mathrm{C} \varepsilon \quad(\mathrm{PLC} \varepsilon)$ is a modular protein that incorporates GEF, PKC and Ras-binding domains (33). Moreover, phospholipase $\mathrm{C} \varepsilon$ could link Ras to calcium $\left(\mathrm{Ca}^{2+}\right)$ mobilization, which has been known to influence cell proliferation and differentiation (34).

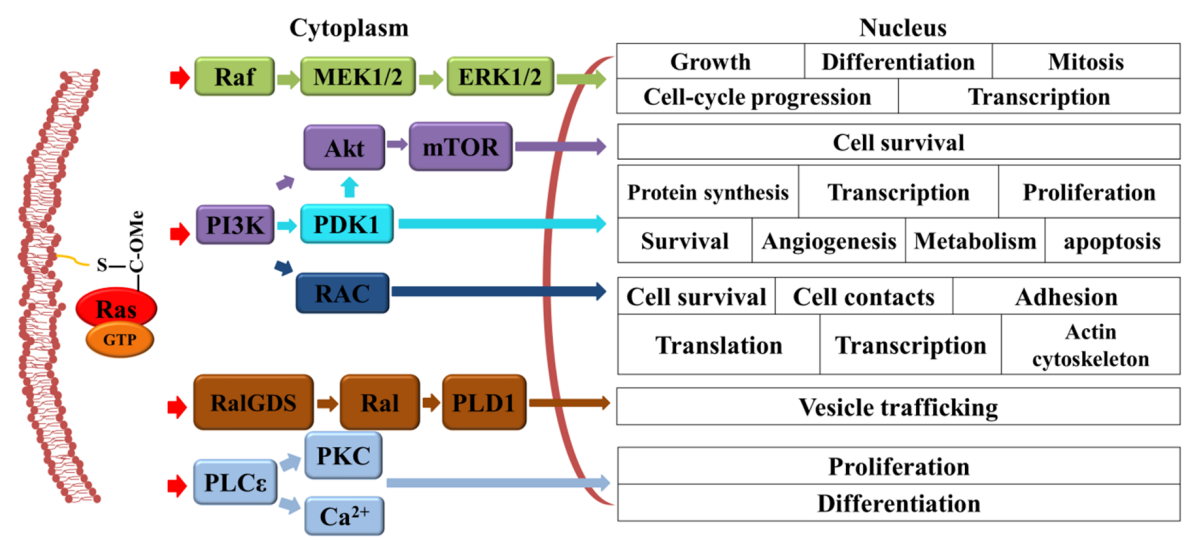

Fig. 2. Regulating signaling downstream of Ras. In the active GTP-bound state, Ras interacts with several families of effector proteins, resulting in stimulation of their catalytic activities. Raf protein kinases activate mitogen-activated protein kinase kinases 1 and 2 (MEK1 and MEK2), which leads to ERK1/2 activation. Phosphoinositide 3-kinases (PI3Ks) generate second-messenger lipids and activate numerous target proteins, including the survival signaling kinase AkT/PDK1. Ras binding activates Ral specific guanine-nucleotide-exchange factors (Ral-GEFs) by targeting them to their substrates, Ral GTPases, which are present in the plasma membrane. Phospholipase C $\varepsilon$ (PLCE) catalyses the activation of protein kinase $\mathrm{C}$ (PKC) and mobilization of calcium from intracellular stores. 


\section{RAS MUTATIONS AND INHIBITORS}

A variety of activating Ras mutations come out in about $30 \%$ of human cancers, and at even higher frequencies in cancers of the pancreas $(90 \%)$, lung $(35 \%)$, thyroid gland $(55 \%)$, colon $(45 \%)$, and liver $(30 \%)(35)$. More than $95 \%$ of Ras mutations are found in codons (amino acids) Gly12, Gly13, or Gln61 (36). These point mutations make the Ras proteins impervious to GTP-induced hydrolysis of GTP to GDP and remain them in the activated form (37). Activating mutations in Ras induce constitutive signaling to downstream targets, i.e., Ras effectors. Many factors identified as Ras effectors are PI3K, RalGDS, RIN1/2, PLC $\varepsilon$, and TIAM1 (38).

Oncogenic mutations, such as Q61, are mainly observed in the K-Ras gene. A total of $15-25 \%$ of lung adenocarcinoma harbors the K-Ras mutation. This peculiarity suggests that each Ras protein plays a distinct role in a tissue-type dependent manner (39). Hence, there may not be a standard Ras-target treatment that applies all Ras mutation (40). Some general strategies for anti-Ras drug development have been suggested, including disruption of regulator/effector interactions, inhibition of membrane associations, downstream effectors, synthetic lethal interactions, and metabolism (41). One of the mutant specific inhibitors reported, SML-8-73-1 (SML), was targeted by the guanine nucleotide binding site of K-Ras G12C mutant (42). The G12C mutant of K-Ras is the principal frequency of K-ras mutations and low rates of causing development of tumors conversion in both $\mathrm{N}$-ras and $\mathrm{H}$-ras. The investigated mutation rate of K-ras in non-small-cell lung cancer (NSCLC) varies from $16 \%$ to $40 \%$ (43). H358 cells with SML-8-73-1 reduces the downstream phosphorylation ratio of ERK and Akt when estimated to medical treatment with negative control, suggesting a compound-dependent effect on K-Ras-driven signaling networks (44). In another study targeted with guanine nucleotide exchange factors (GEFs), SOS1 converts Ras from a GDP-bound to a GTP-bound state (45). The Ras-SOS1 complex was shown to have an $\alpha$-helix of SOS1 that binds to a pocket located between the SI and SII regions of K-Ras (46). DCAI and HBS3 peptides are designed to inhibit SOS1 mediated nucleotide exchange by obstructing the molecular interaction between Ras and SOS1, which inhibits activation of Ras in cells $(47,48)$. However, like numerous other known inhibitors, the GEF inhibitors reportedly interact weakly to $\mathrm{K}-\mathrm{Ras}$, and the development of new analogues with improved affinity will be a very difficult work. In another study, farnesyltransferase (FTase) inhibitors (FTIs) and geranyleranyltransferase I (GGTase I) inhibited the plasma membrane fusion and subcellular location of Ras (49). These compounds were shown to be a powerful treatment for $\mathrm{H}$-Ras mutation cancers. However, the H-Ras mutant frequency is low and the compounds have been shown to exert serious toxicity in normal tissues.

To date, directly blocking oncogenic Ras activity has been a challenging and unsuccessful endeavor. Therefore, past studies have targeted effector pathways downstream of Ras-mediated oncogenesis (50). The pathways of Raf-MEK-ERK and PI3K-Akt are the best-characterized Ras effector pathways, initiating cascades of protein-protein interactions that may lead to cell proliferation (51). Sorafenib is an initial antitumor drug that targets Raf kinases to be developed (52). This inhibits the activity of several tyrosine kinases associated with tumor angiogenesis and progression, incorporating the vascular endothelial growth factor receptor (VEGFR) family (53).

One of the clear manners is the use of ERK inhibitors because the mechanism of resistance to Raf inhibitors is frequently due to the phosphorylation of ERK. The inhibitors targeted that ATP-bound pocket of ERK acts in competition with ATP (54). However, those inhibitors obstruct ERK feedback phosphorylation and inactivation of Raf, which causes improved MEK phosphorylation. Although effector pathways inhibition seems to be the most promising Ras-targeted strategy, considerable challenges remain.

Other biological studies have pointed that unusual activation of the Ras-Raf-MEK-ERK pathway often results in hepatocellular carcinoma (HCC). The second-generation allosteric non-ATP competitive MEK1/2 inhibitor selumetinib is a benzimidazole derivative that has been shown to contribute to the inhibition of ERK1/2 phosphorylation (55). Selumetinib is well tolerated, but not ideal for treatment of advanced HCC. Recent studies have shown that salinomycin high specific inhibits the K-Ras activation. Salinomycin effectively decreases effector into K-Ras and then at least impairs the Ras/MAPK signaling pathway and cell proliferation (56). Inhibition of effector pathways has offered a new way of RAS-targeted strategy, and therefore the efforts on the identification and characterization of new Ras effectors will allow us to have better chance for anti-cancer drug discovery.

\section{CONCLUSIONS}

Fourth decade research in oncogenic Ras, has generated thus far serves as a rich and instructive backdrop for the questions that lie ahead. Nevertheless, we still have a great deal to learn about these cancers before we can confidently treat them effectively. Changes in cellular metabolism by Ras have recently been identified. In the future, many studies will talk about whether these changes could be utilized for future therapeutic approach. Moreover, in 2014, the United States National Cancer Institute triggered the Ras Initiative, which costs $\$ 10$ million annually to identify new therapies to treat Ras-induced cancers (57). In addition research groups has discovered chemicals that can produce the first inhibitor that targets the mutant Ras protein. Ras target treatment in human cancer is still a significant challenge. A wealth of knowledge acquired through experiments will play an important role in promotion of new drug development. In summary, critical assessment of past efforts coupled with discussions of biochemical properties will enable the development of more 
effective cancer therapies.

\section{ACKNOWLEDGEMENTS}

This study was supported by the Basic Science Research Program through the National Research Foundation of Korea (NRF) funded by the Ministry of Education, Science and Technology (2015R1D1A1A01059594) to S.B.J. and (2016R 1D1A1B02011142) to J.M.S.

\section{CONFLICTS OF INTEREST}

The authors have no conflicting financial interests.

\section{REFERENCES}

1. Cox AD and Der CJ (2010) Ras history: The saga continues. Small GTPases 1, 2-27

2. Victor T, Du Toit R, Jordaan AM, Bester AJ and Van Helden PD (1990) No evidence for point mutations in codons 12, 13, and 61 of the ras gene in a high-incidence area for esophageal and gastric cancers. Cancer Res 50, 4911-4914

3. Chang YS, Yeh KT, Hsu NC et al (2010) Detection of N-, $\mathrm{H}-$, and KRAS codons 12, 13, and 61 mutations with universal RAS primer multiplex PCR and $\mathrm{N}-, \mathrm{H}-$, and KRAS-specific primer extension. Clin Biochem 43, 296-301

4. Berns A (2008) Kras and Hras - what is the difference? Nat Genet 40, 1149-1150

5. Eungdamrong NJ and lyengar R (2004) Computational approaches for modeling regulatory cellular networks. Trends Cell Biol 14, 661-669

6. Brock EJ, Ji K, Reiners JJ and Mattingly RR (2016) How to target activated Ras proteins: direct inhibition vs. induced mislocalization. Mini Rev Med Chem 16, 358-369

7. Milburn MV, Tong L, Brunger A and Yamaizumi Z (1990) Molecular switch for signal transduction: structural differences between active and inactive forms of protooncogenic ras proteins. Science 247, 939-945

8. De Luca A, Maiello MR, D'Alessio A, Pergameno M and Normanno $N$ (2012) The RAS/RAF/MEK/ERK and the PI3K/AKT signalling pathways: role in cancer pathogenesis and implications for therapeutic approaches. Expert Opin Ther Targets 16, 17-27

9. Bos JL (1997) Ras-like GTPases. Biochim Biophys Acta $1333,19-31$

10. Touchot $N$, Chardin $P$ and Tavitian A (1987) Four additional members of the ras gene superfamily isolated by an oligonucleotide strategy: molecular cloning of YPT-related cDNAs from a rat brain library. Proc Natl Acad Sci U S A 84, 8210-8214

11. Wittinghofer A and Vetter IR (2011) Structure-function relationships of the $\mathrm{G}$ domain, a canonical switch motif. Annu Rev Biochem 80, 943-971

12. Ostrem JM, Peters U, Sos ML, Wells JA and Shokat KM (2013) K-Ras (G12C) inhibitors allosterically control GTP affinity and effector interactions. Nature 503, 548-551
13. Prior IA, Lewis PD and Mattos C (2012) A comprehensive survey of Ras mutations in cancer. Cancer Res 72, 2457-2467

14. Reiss Y, Goldstein JL, Seabra MC, Casey PJ and Brown MS (1990) Inhibition of purified p21ras farnesyl: protein transferase by Cys-AAX tetrapeptides. Cell 62, 81-88

15. Michaelson D, Ali W, Chiu VK et al (2005) Postprenylation CAAX processing is required for proper localization of Ras but not Rho GTPases. Mol Biol Cell 16, 1606-1616

16. Manolaridis I, Kulkarni K, Dodd RB et al (2013) Mechanism of farnesylated CAAX protein processing by the intramembrane protease Rce1. Nature 504, 301-305

17. Hancock JF and Robert G (2005) Ras plasma membrane signalling platforms. Biochem J 389, 1-11

18. Smith MJ and Ikura M (2014) Integrated RAS signaling defined by parallel NMR detection of effectors and regulators. Nat Chem Biol 10, 223-230

19. Scheffzek K, Ahmadian MR, Kabsch W et al (1997) The Ras-RasGAP complex: structural basis for GTPase activation and its loss in oncogenic Ras mutants. Science 277, 333-339

20. Feig LA (1999) Tools of the trade: use of dominantinhibitory mutants of Ras-family GTPases. Nat Cell Biol 1, 25-27

21. Roberts PJ and Der CJ (2007) Targeting the Raf-MEK-ERK mitogen-activated protein kinase cascade for the treatment of cancer. Oncogene 26, 3291-3310

22. Leicht DT, Balan V, Kaplun A et al (2007) Raf kinases: function, regulation and role in human cancer. Biochim Biophys Acta 1773, 1196-1212

23. Downward J (2003) Targeting RAS signalling pathways in cancer therapy. Nat Rev Cancer 3, 11-22

24. Manna PR and Stocco DM (2011) The role of specific mitogen-activated protein kinase signaling cascades in the regulation of steroidogenesis. J Signal Transduct 2011, 821615

25. Zhang W and Liu HT (2002) MAPK signal pathways in the regulation of cell proliferation in mammalian cells. Cell Res 12, 9-18

26. Wong KK, Engelman JA and Cantley LC (2010) Targeting the $\mathrm{PI} 3 \mathrm{~K}$ signaling pathway in cancer. Curr Opin Genet Dev 20, 87-90

27. Eser S, Reiff N, Messer $M$ et al (2013) Selective requirement of PI3K/PDK1 signaling for Kras oncogene-driven pancreatic cell plasticity and cancer. Cancer Cell 23, 406-420

28. Pearce LR, Komander D and Alessi DR (2010) The nuts and bolts of AGC protein kinases. Nat Rev Mol Cell Biol $11,9-22$

29. Sadeghi N and Gerber DE (2012) Targeting the PI3K pathway for cancer therapy. Future Med Chem 4, 1153-1169

30. Welch HC, Coadwell WJ, Stephens LR and Hawkins PT (2003) Phosphoinositide 3-kinase-dependent activation of Rac. FEBS Lett 546, 93-97

31. Hao $Y$, Wong $R$ and Feig LA (2008) RalGDS couples growth factor signaling to Akt activation. Mol Cell Biol 28, 2851-2859

32. Kashatus DF (2013) Ral GTPases in tumorigenesis: emerging from the shadows. Exp Cell Res 319, 2337-2342 
33. Bunney TD and Katan M (2006) Phospholipase C epsilon: linking second messengers and small GTPases. Trends Cell Biol 16, 640-648

34. Urtreger AJ, Kazanietz MG and Bal de Kier Joffé ED (2012) Contribution of individual PKC isoforms to breast cancer progression. IUBMB Life 64, 18-26

35. Leshchiner ES, Parkhitko A, Bird GH et al (2015) Direct inhibition of oncogenic KRAS by hydrocarbon-stapled SOS1 helices. Proc Natl Acad Sci U S A 112, 1761-1766

36. Aviel-Ronen S, Blackhall FH, Shepherd FA and Tsao MS (2006) K-ras mutations in non-small-cell lung carcinoma: a review. Clin Lung Cancer 8, 30-38

37. Chang YS, Yeh KT, Hsu NC et al (2010) Detection of N-, $\mathrm{H}-$, and KRAS codons 12, 13, and 61 mutations with universal RAS primer multiplex PCR and $\mathrm{N}-, \mathrm{H}-$, and KRAS-specific primer extension. Clin Biochem 43, 296301

38. Repasky GA, Chenette EJ and Der CJ (2004) Renewing the conspiracy theory debate: does Raf function alone to mediate Ras oncogenesis? Trends Cell Biol 14, 639-647

39. Westcott PM and To MD (2013) The genetics and biology of KRAS in lung cancer. Chin J Cancer 32, 63-70

40. Cox AD, Fesik SW, Kimmelman AC, Luo J and Der CJ (2014) Drugging the undruggable RAS: mission possible? Nat Rev Drug Discov 13, 828-851

41. Papke B and Der CJ (2017) Drugging RAS: Know the enemy. Science 355, 1158-1163

42. Hunter JC, Gurbani D, Ficarro SB et al (2014) In situ selectivity profiling and crystal structure of SML-8-73-1, an active site inhibitor of oncogenic K-Ras G12C. Proc Natl Acad Sci U S A 111, 8895-8900

43. Boch C, Kollmeier J, Roth A et al (2013). The frequency of EGFR and KRAS mutations in non-small cell lung cancer (NSCLC): routine screening data for central Europe from a cohort study. BMJ Open 3, e002560

44. Lim SM, Westover KD, Ficarro SB et al (2014) Therapeutic Targeting of Oncogenic K-Ras by a Covalent Catalytic Site Inhibitor. Angew Chem Int Ed Engl 53, 199-204

45. Bos JL, Rehmann $\mathrm{H}$ and Wittinghofer A (2007) GEFs and GAPs: critical elements in the control of small $G$ proteins. Cell 129, 865-877
46. Winter JJ, Anderson M, Blades K et al (2015) Small molecule binding sites on the Ras: SOS complex can be exploited for inhibition of Ras activation. J Med Chem 58, 2265-2274

47. Maurer T, Garrenton LS, Oh A et al (2012) Smallmolecule ligands bind to a distinct pocket in Ras and inhibit SOS-mediated nucleotide exchange activity. Proc Natl Acad Sci U S A 109, 5299-5304

48. Patgiri A, Yadav KK, Arora PS and Bar-Sagi D (2011) An orthosteric inhibitor of the Ras-Sos interaction. Nat Chem Biol 7, 585-587

49. Capell BC, Erdos MR, Madigan JP et al (2005) Inhibiting farnesylation of progerin prevents the characteristic nuclear blebbing of Hutchinson-Gilford progeria syndrome. Proc Natl Acad Sci U S A 102, 12879-12884

50. Baker NM and Der CJ (2013) Cancer: Drug for an 'undruggable' protein. Nature 497, 577-578

51. Steelman LS, Chappell WH, Abrams SL et al (2011) Roles of the Raf/MEK/ERK and PI3K/PTEN/Akt/mTOR pathways in controlling growth and sensitivity to therapy-implications for cancer and aging. Aging (Albany NY) 3, 192-222

52. Cervello M, Bachvarov D, Lampiasi N et al (2012) Molecular mechanisms of sorafenib action in liver cancer cells. Cell Cycle 11, 2843-2855

53. Samant RS and Shevde LA (2011) Recent advances in anti-angiogenic therapy of cancer. Oncotarget 2, 122-134

54. Aronov AM, Tang Q, Martinez-Botella G et al (2009) Structure-guided design of potent and selective pyrimidylpyrrole inhibitors of extracellular signal-regulated kinase (ERK) using conformational control. J Med Chem $52,6362-6368$

55. Do K, Speranza G, Bishop R et al (2015) Biomarker-driven phase 2 study of MK-2206 and selumetinib (AZD6244, ARRY-142886) in patients with colorectal cancer. Invest New Drugs 33, 720-728

56. Najumudeen AK, Jaiswal A, Lectez B et al (2016) Cancer stem cell drugs target K-ras signaling in a stemness context. Oncogene 35, 5248-5262

57. Ledford $\mathrm{H}$ (2015) Cancer: The ras renaissance. Nature 520, 278-280 